\title{
LONGITUDINAL ELECTRODE VOLTAGE DISTRIBUTION ON A 4-ROD RFQ SIMULATION MODEL*
}

\author{
P. Fischer ${ }^{\#}$, N. Mueller, A. Schempp, J.-W.-Goethe University, Frankfurt, Germany
}

\section{Abstract}

For a proper working RFQ with minimized particle losses, a constant longitudinal voltage distribution on the electrodes is needed [1]. This assures a sufficient compensation of the beam space change. The local electrode voltage and its change is mainly given by the varying aperture of the quadrupole and in consequence the changing local capacity. The simulation of the RFQ model with modulated electrodes is a very complex thread. An example for such a model will be given here.

\section{SIMULATION OF 4-ROD RFQS}

The rf simulation of 4-rod $\lambda / 2$ RFQ accelerators is usually done with CST Microwave Studio, a software that employs finite integration method to calculate the eigenmodes and the field distribution of the resonant structure.

The geometrical layout of the resonant structure requires a precise determination of the eigenmodes, also the field distribution of the structure has to be realistic, deviations can cause particle losses.

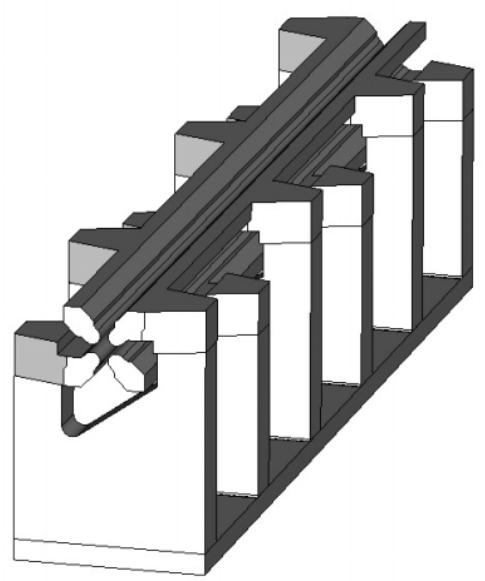

Figure 1: A 4-rod N/2 RFQ simulation model with six stems and plain electrodes.

\section{FIELD DISTRIBUTION}

To get a field distribution as used, beam dynamics simulations, the electrode voltage has to be constant in longitudinal direction along the resonant structure. If the local voltage is too low, particles get lost due to weak focussing forces, as can be seen in figure 2 . In this example, the electrode voltage was reduced to $70 \%$ in a

\footnotetext{
*Work supported by BMBF

\#P.Fischer@iap.uni-frankfurt.de
}

small range of some $\mathrm{cm}$. This point acts like a bottleneck to the beam, the number of lost particles is defined by the weakest point of the structure. Transmission was reduced there from $\mathrm{T}_{\text {flat }}=99 \%$ to $\mathrm{T}_{\text {bottleneck }}=85 \%$.
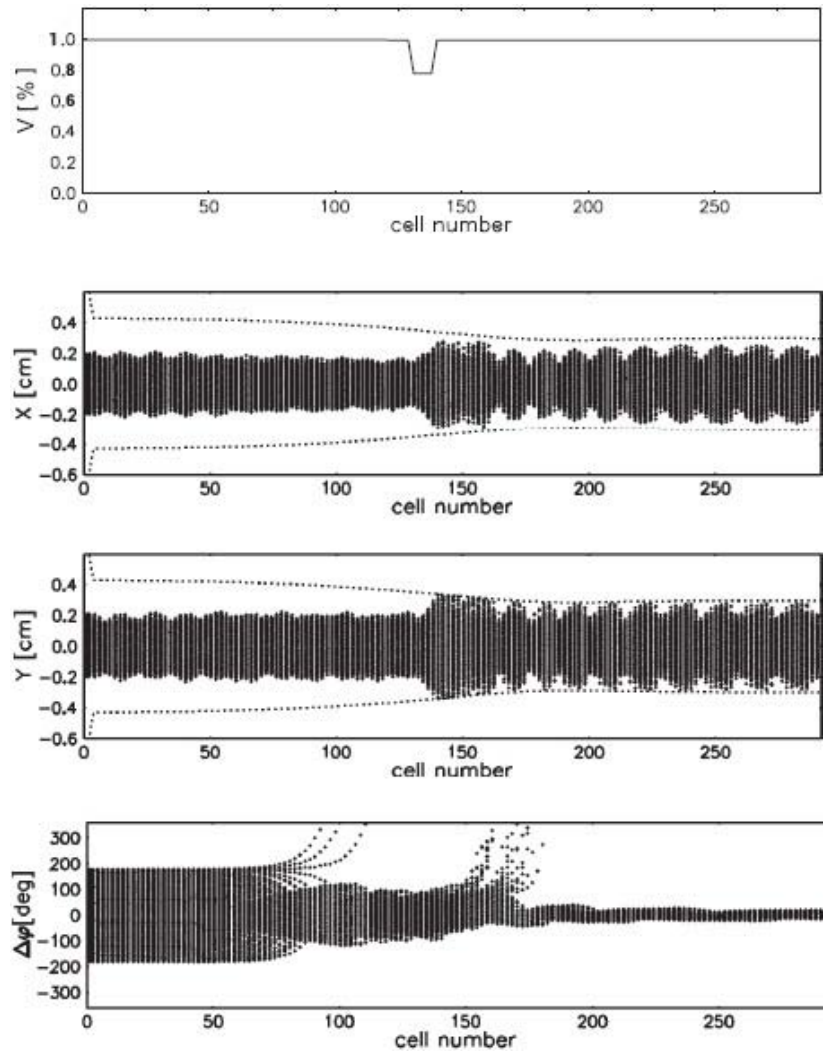

Figure 2: Beam dynamics simulation of a RFQ model with insufficient focussing forces.

In RFQ simulation models, unmodulated rods or vanes are taken as electrodes. Then, the capacity of the resonant structure, which is given by the electrodes, is nearly constant.

In real RFQ structures, the aperture and modulation changes with the longitudinal position and thus the capacity. In consequence, the longitudinal field distribution is not constant, which can be measured by a perturbation measurement.

\section{ADVANCED SIMULATIONS}

The beam dynamics simulation example in the last section showed, that a constant longitudinal electrode voltage distribution is important for a proper working machine. Thus, varying capacity in terms of the changing aperture should also be in the simulations. Because of the big difference between the electrodes length of about two 
to four meters and the modulation geometry in range of few $1 / 100 \mathrm{~mm}$, a simulation model gets very complex and the effort of calculation it requires gets very big.

Thus, a simulation model was generated containing the full electrode modulation data. As a first step, triangles were taken instead of a sinusoidal modulation. This can be seen in figure 3 .

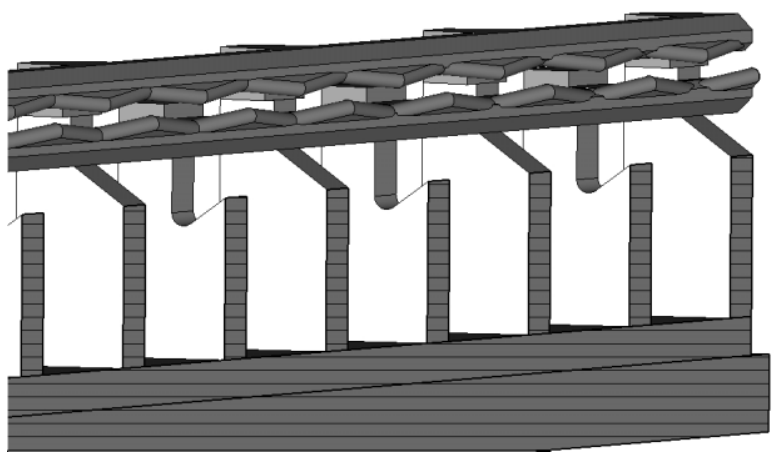

Figure 3: Vertical cross-section in beam direction of a RFQ model with modulated electrodes.

The simulation with triangles as approximation shows a completely different field distribution compared with unmodulated electrodes. The distribution is strongly tilted, the high-energy end has very low field strength. The result is drawn in figure 4 (peaks are calculation errors).

In a second step, electrodes with a sinusoidal modulation were simulated. Less calculation errors occurred and comparing to measured values, the distribution became more realistic, figure 4 shows the distribution graph. It was also possible to get a result of the accelerating field component $\mathrm{E}_{\mathrm{z}}$, which only can be seen in a simulation model with modulated electrodes (see figure 5).

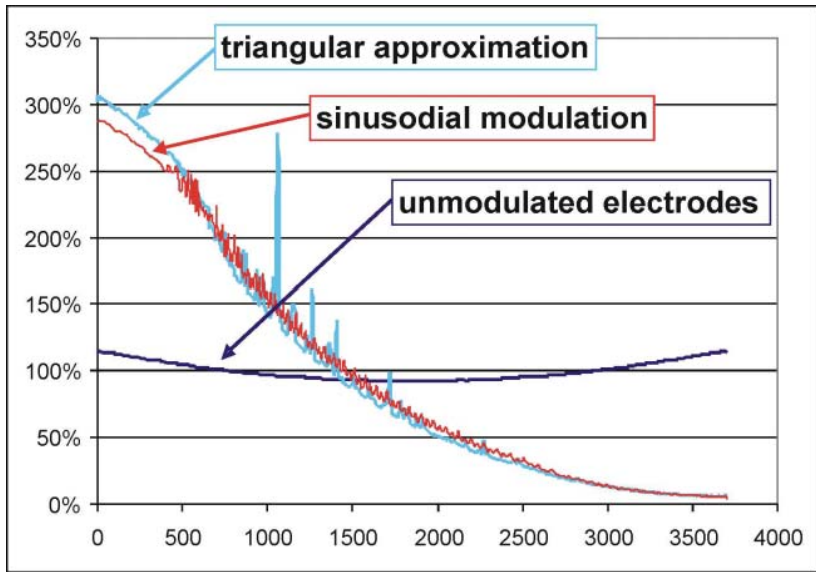

Figure 4: Longitudinal quadrupole field distribution of different simulation models.

\section{CONCLUSIONS}

A simulation of a 4-rod RFQ structure with very detailed electrode geometry has been done with Microwave Studio, and the simulation result, the longitudinal field distribution was extracted. Next steps are the rf tuning of the structure with tuning plates.

\section{REFERENCES}

[1] P. Fischer, A. Schempp: "TUNING OF A 4-ROD CW-MODE RFQ ACCELERATOR", EPAC'06, June2006, Edinburgh, Scotland, UK, p.1583.

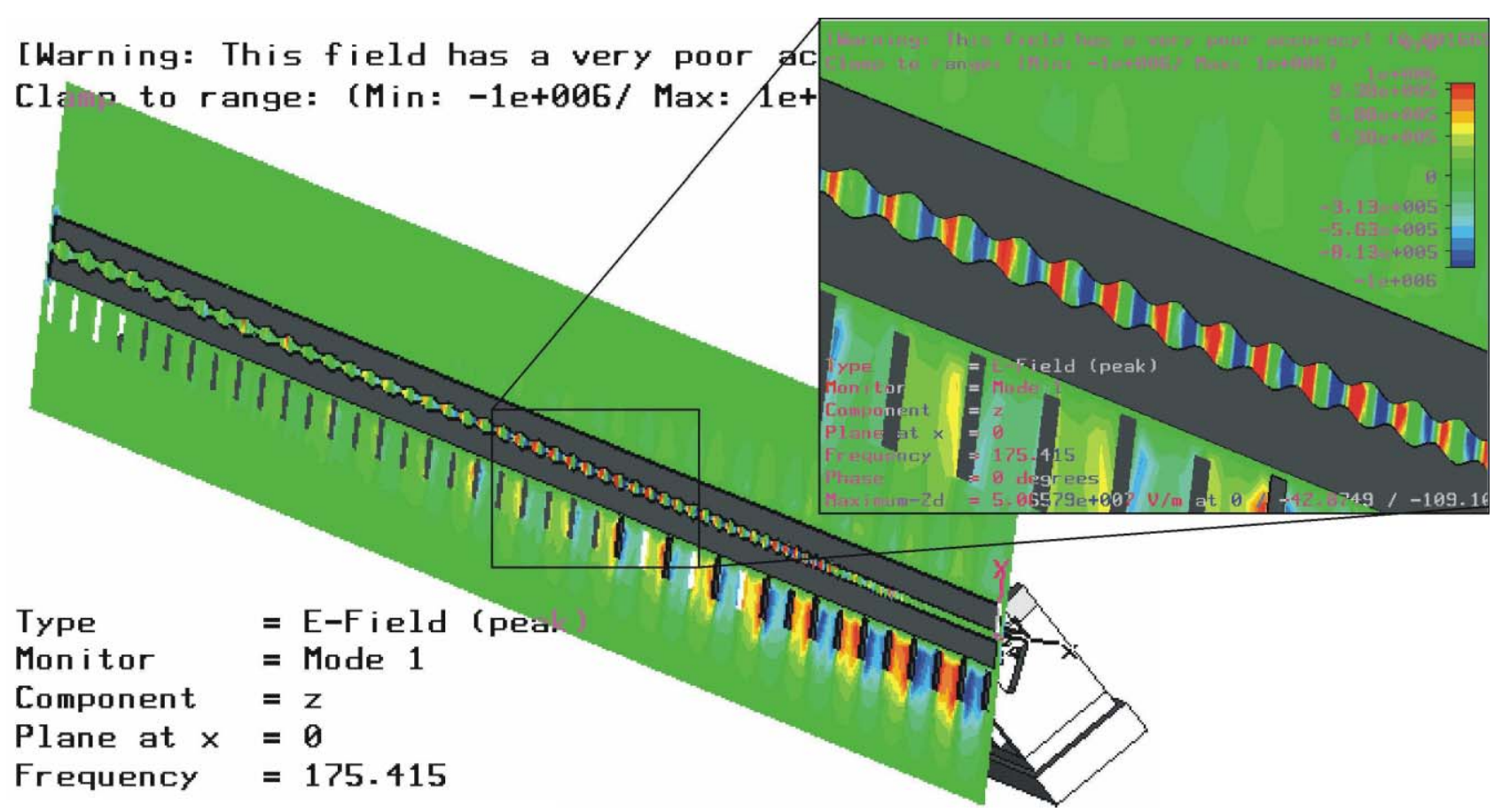

Figure 5: Distribution of the accelerating field $\mathrm{E}_{\mathrm{z}}$. 